\title{
PROPUESTA DE UN ÍNDICE PARA LA MEDICIÓN DE LA CALIDAD DE VIDA EN COSTA RICA
}

\author{
Hazel Brenes* \\ Edgar E. Gutiérrez-Espeleta**
}

RESUMEN

Con el propósito de aproximar una evaluación de la calidad de vida de los y las habitantes de nuestro país se construyeron varios índices a partir de estadísticas continuas, para el período 1990-2003. Se utilizaron dos métodos de agregación para obtener puntuaciones anuales de los índices. Además de la calificación global de calidad de vida, los métodos propuestos permitieron obtener puntuaciones para cada una de sus dimensiones. Los índices aquí presentados son sólo una propuesta preliminar debido a la poca disponibilidad de datos confiables para la medición de todas las dimensiones establecidas y además por la falta de evaluación por parte de expertos. No obstante, sus resultados permiten ver que, desde el año 1990 hasta el 2003, la calidad de vida ha alcanzado niveles regulares, $y$ su tendencia es al deterioro.

PALABRAS CLAVE: CALIDAD DE VIDA * CLASES EVALUATIVAS * ÍNDICES * INDICADORES

\section{ABSTRACT}

With the intention to approximate an assessment of quality of life of inhabitants in our country, indices were developed from continuous statistics, for the period 1990 to 2003. Two aggregation methods were utilized to obtain annual marks for the indices. Beyond the global score for quality of life, scores for each dimension used in developing the index were obtained. These indices are just a proposal because of the lack of data availability and peer discussions on this subject. Nevertheless, results allow seeing that from 1990 to 2003, quality of life has reached medium levels with a trend to deterioration.

KEY WORDS: QUALITY OF LIFE * EVALUATION CLASSES * INDICES * INDICATORS

\section{JUSTIFICACIÓN}

La idea de dar seguimiento a la calidad de vida de las y los habitantes de Costa Rica es

Escuela de Estadística, Universidad de Costa Rica hazel_brenes@yahoo.com social y académicamente relevante. Desde el punto de vista social, la calidad de vida ayuda a engarzar las tendencias macro del desarrollo con la vida de las personas. Desde el punto de 
vista académico, puede contribuir al desarrollo de nuevas investigaciones, sistemas de información y evaluaciones más precisas dentro de la sociedad costarricense (PEN, 2000).

El concepto calidad de vida tiene significados complejos $y$ variados, por lo que su medición puede ser un reto. Esto se debe fundamentalmente a la dificultad de captar todas las dimensiones que encierra el concepto. Para lograr este objetivo, es necesaria la utilización de varios indicadores. Con el fin de derivar una sola cifra a partir de un conjunto de indicadores, los analistas de datos cuantitativos han concebido técnicas para combinarlos. Dentro de estas técnicas se encuentran los índices, los cuales resumen varios indicadores en una sola puntuación numérica, al mismo tiempo que, muchas veces, conservan los detalles específicos de cada indicador (Babbie, 1999).

Puede decirse que, de la misma manera que una persona encargada de tomar decisiones solicita un resumen ejecutivo cuando le presentan un reporte de cien páginas, los índices resumen realidades complejas, ya que su función es dar una idea clara y precisa de una situación concreta en forma rápida, sencilla, visual y abreviada.

De acuerdo con Gutiérrez (1994), el uso de indicadores permite entender las tendencias del fenómeno bajo estudio, mejorar cualitativamente el proceso de retroalimentación de los que toman las decisiones, educar al público sobre situaciones particulares y obtener una perspectiva holística o integral del objeto de análisis. Como estadísticos que son, permiten reducir la dimensionalidad del problema, haciendo posible una mayor capacidad de interpretación y conceptualización de problemas específicos.

Una propuesta sobre la medición de la calidad de vida en el país, por medio de un índice, provee a los tomadores de decisiones, y la comunidad en general, de una herramienta que comunica información relevante $y$ transparente, relacionada con la calidad de vida.

Este artículo se encuentra organizado en cuatro secciones. En la segunda sección, se presenta la metodología utilizada para construir a partir de estadísticas continuas un Índice de calidad de vida. La tercera parte de este documento corresponde a la presentación y discusión de los resultados obtenidos al aplicar la metodología descrita anteriormente. Finalmente se presentan las principales conclusiones de la investigación.

\section{MÉTODOS Y MATERIALES}

\subsection{ESQUEMA PARA LA CONSTRUCCIÓN DE ÍNDICES}

Con el fin de alcanzar el objetivo propuesto inicialmente, es decir la construcción de un índice de calidad de vida, se adoptó el esquema propuesto por Gutiérrez (1998) y cuyos pasos se muestran a continuación:

FIGURA 1

PASOS EN LA CONSTRUCCIÓN DE INDICADORES

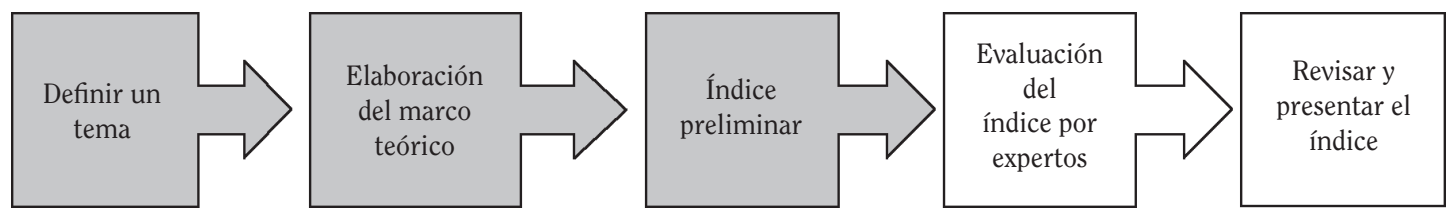

Cabe destacar que este proyecto se centró en los tres primeros pasos. Como primer paso, el tema definido fue la evaluación en forma objetiva y aproximada de la calidad de vida. Segundo, desarrolla el marco teórico.
El tercer paso consistió en compilar los datos de acuerdo con las dimensiones identificadas en el marco teórico y las fuentes de información existentes. Y el cuarto, las variables fueron agregadas en correspondencia con 
el marco conceptual, obteniendo indicadores o índices preliminares.

Los últimos dos pasos consisten en la evaluación del índice por un grupo de expertos con el fin de garantizar tanto la calidad cuantitativa como cualitativa del índice, posteriormente el índice revisado es presentado al grupo de usuarios potenciales tanto para una revisión final, como para garantizar su utilidad y transparencia al comunicar información relevante para la toma de decisión. Finalmente, el índice es utilizado por los diferentes segmentos de la población.

\subsubsection{ELABORACIÓN DEL MARCO CONCEPTUAL}

Para el desarrollo de este proyecto calidad de vida se definió como el conjunto de condiciones que contribuyen a hacer agradable y valiosa la vida. Dichas condiciones están referidas a vivir en forma libre, digna, segura, creativa y solidaria. Cabe destacar que esta definición es el resultado de la consulta bibliográfica de los principales enfoques empleados en las últimas décadas para evaluar la calidad de vida (Brenes, 2004).

La dimensión libertad, está referida a la necesidad que tienen las personas de poder desarrollar sus puntos de vista, tener autonomía personal sin interferencia del Estado, poder participar libremente en la escogencia de las autoridades e influir en las decisiones públicas que afectan a las comunidades nacionales, regionales o locales (FH, 2003). Según Nussbaum (1999), los derechos políticos no son sólo importantes para satisfacer necesidades, sino también son decisivos para poder formularlas.

La dignidad en este caso está relacionada al derecho de las personas a tener un trabajo justo $y$ adecuadamente retribuido, bajo condiciones de seguridad e higiene ocupacional, tener oportunidades para la realización y el crecimiento personal, vivir en un medio ambiente seguro y como lo estableció la Organización Mundial de la Salud, (OMS) desde 1960, las personas deben poder gozar de un estado general de bienestar físico, mental y social, $y$ no meramente no tener enfermedades o dolencias (van Poll, 1997). Una persona que no goce de plena seguridad (económica, personal, jurídica y ciudadana), tampoco puede tener una buena calidad de vida.

Según los resultados de la Encuesta de Calidad de vida de Unimer RI (2002), 95,5\% de las personas encuestadas estuvieron de acuerdo con que la calidad de vida es "contar con un buen balance de tiempo para el trabajo, el amor, la recreación, el estudio, el pensamiento y el ejercicio". Para que esto pueda ser una realidad las personas deben tener acceso a eventos de entretenimiento, deportivos, artes visuales y recibir estímulos para desarrollar su capacidad de invención y creación. Estos elementos son los que se asocian a la dimensión creatividad.

El último aspecto de la calidad de vida está relacionado con la necesidad de todos y para todos de contribuir con los otros seres humanos, es decir la solidaridad que exista entre los habitantes de un país.

Utilizando el ejercicio grupal realizado por el PEN (2000) y el método citado en Gutiérrez (1998) se puede plantear:

El método aplicado para el establecimiento de este marco conceptual fue deductivo, es decir, primero se desagregó el concepto de calidad de vida en cinco dimensiones cada una de las cuales fue a su vez dividida en temas y como último paso se buscaron indicadores para medir cada uno de estos temas. Por otra parte, los métodos de agregación descritos en la siguiente sección, obedecen a un razonamiento inductivo, en el cual se agregó información específica (indicadores) y se obtuvieron puntuaciones para cada una de las dimensiones, las cuales a su vez fueron agregadas para obtener la calificación asignada a la calidad de vida. 
FIGURA 2

\section{DEFINICIÓN OPERATIVA DE CALIDAD DE VIDA}

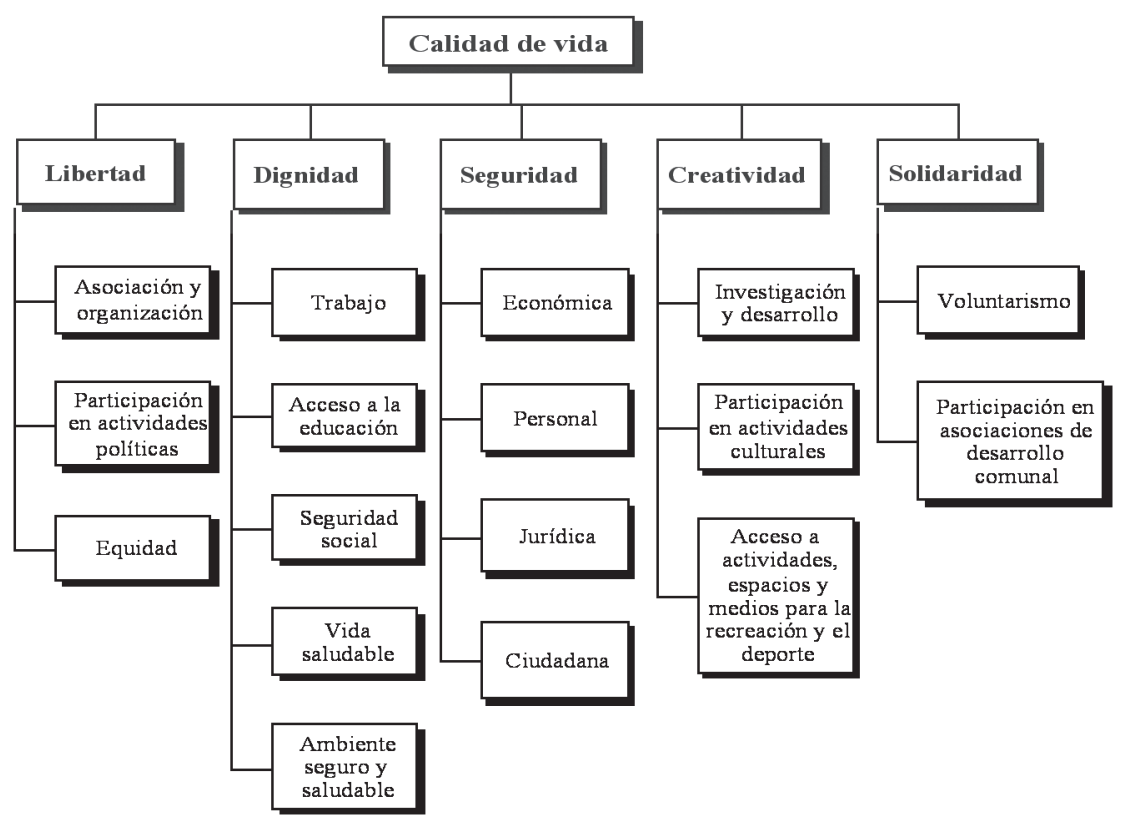

\subsubsection{CONSTRUCCIÓN DEL ÍNDICE PRELIMINAR}

\subsection{SELECCIÓN DE INDICADORES}

Una vez que se estableció la definición operativa de calidad de vida, se procedió a revisar y consultar la bibliografía disponible no sólo en Costa Rica, sino también en el resto del mundo, con el fin de elaborar una primera propuesta de indicadores viables $y$ pertinentes al tema de estudio.

Entre los criterios utilizados para la selección de los indicadores se encontraron la importancia de la variable en la medición de la calidad de vida, la calidad de los datos, el interés de los políticos y ciudadanos por conocer de esta variable, la información adicional que aportaba la variable sobre la calidad de vida en relación con otras variables y además que existieran registros de esta variable para el período de estudio (1990 hasta 2003).

Uno de los principales criterios en la recolección de los datos fue la utilización de fuentes primarias, esto con el fin de evitar errores de trascripción por parte de fuentes secundarias y facilitar la obtención de las definiciones y la metodología empleada para la recolección de los datos, además en lo posible se utilizaron datos oficiales.

\subsubsection{MÉTODOS DE AGREGACIÓN}

^ Método de máximos y mínimos

El primer método de agregación aplicado consistió en la asignación de metas para cada indicador y su calificación de 0 a 1 según la observación se acercara o se alejara del valor deseado. Esta transformación se realizó en forma análoga a la utilizada por el Programa de las Naciones Unidas para el Desarrollo (PNUD) para el cálculo del Índice de Desarrollo Humano (IDH).

a. Cada observación de la variable (valor $x_{i}$ efectivo) se transformó mediante la siguiente fórmula: 
CASO FÓRMULA

1. $\quad x^{*}=\frac{\text { Valor } x_{i} \text { efectivo }- \text { valor } x_{i} \text { mínimo }}{\text { Valor } x_{i} \text { máximo }- \text { valor } x_{i} \text { mínimo }}$

Si $x_{i}$ efectivo $<x_{i}$ mínimo $\Rightarrow x^{*}=0$

Si $x_{i}$ efectivo $>x_{i}$ máximo $\Rightarrow x^{*}=1$

2. $\quad x^{*}=\frac{\text { Valor } x_{i} \text { máximo }- \text { valor } x_{i} \text { efectivo }}{\text { Valor } x_{i} \text { máximo }- \text { valor } x_{i} \text { mínimo }}$

Si $x_{i}$ efectivo $<x_{i}$ mínimo $\Rightarrow x^{*}=1$

Si $x_{i}$ efectivo $>x_{i}$ máximo $\Rightarrow x^{*}=0$

Los valores máximos y mínimos se determinaron de acuerdo con metas fijadas para cada indicador. Para fijar las metas (o valores deseados) se utilizaron diferentes estrategias: en algunos casos con base en los niveles promedio de países que se consideran como "modelo", en otros casos las metas fueron fijadas según la distribución del indicador en el ámbito mundial tomando en cuenta la distribución según percentiles, otra opción fue con base en la tendencia del indicador $y$ en algunos otros casos se tomaron metas previamente construidas por González et al. (1999) para el Índice Aproximado de Sostenibilidad (IAS) (en el anexo A se muestran los valores mínimos y máximos fijados para cada indicador).

Por ejemplo, para el quinquenio 20002005, la Tasa de Mortalidad Infantil (TMI), para Finlandia se estima en 4, mientras que la correspondiente a Suecia se estima en 3,4 (UNSD, 2003), en este caso el valor mínimo fue fijado en 4 . Por otra parte, diversas Conferencias establecieron un valor meta de 50 para la TMI para el año 2000, por lo que esta cifra fue tomada como valor máximo. Considerando que en el año 2002, el Centro Centroamericano de Población (CCP-UCR) (2004) reportó una TMI para Costa Rica igual a $10,25, x^{*}$ se determinó de la siguiente manera:

$$
x^{*}=\frac{50-10,25}{50-4}=0,864
$$

Se utiliza si:

A mayor valor de la variable se supone una mayor calidad de vida (por ejemplo, esperanza de vida)

ó

A menor valor de la variable se supone una mayor calidad de vida (por ejemplo, tasa de mortalidad infantil)

b. Para cada dimensión se combinaron los $\mathrm{x}^{*}$ mediante promedios simples. Aunque el objetivo final era obtener un índice general de calidad de vida (ICV), el cálculo de índices por dimensión enriqueció el análisis del fenómeno de estudio.

c. Finalmente, los índices correspondientes a cada dimensión se promediaron ponderados por el número de indicadores utilizados para su cálculo para obtener el ICV. Matemáticamente esto es equivalente a calcular un promedio simple de todos los indicadores sin hacer distinciones por dimensión.

$\diamond$ Método de clases evaluativas

La segunda forma de agregación se basó en el método propuesto por Gutiérrez (1994) para el cálculo de su Índice Aproximado de Sostenibilidad, que consiste en lo siguiente:

a. Cada indicador fue calificado utilizando una escala de cinco puntos $(+2,+1,0$, $1,-2)$ según clases evaluativas propuestas. Por ejemplo, con el fin de calcular el IAS, González et al. (1999) presentó las siguientes categorías para la Tasa de Mortalidad Infantil (por mil nacidos vivos): 
El criterio principal para la definición de los límites de las clases fue la distribución de cada indicador con respecto a las cifras de diferentes países a escala mundial, por ejemplo, se asignó una puntuación de +2 cuando el valor del indicador se ubicaba entre el 5\% de los paí- ses mejor posicionados, o bien se asignó a la categoría -2 cuando el país estaba ubicado dentro del $50 \%$ con un peor posicionamiento (los límites fijados para las clases evaluativas de desempeño se muestran en el anexo B ).

\begin{tabular}{ccccc}
\hline+2 & +1 & 0 & -1 & -2 \\
\hline Menor o igual a 7 & De más de 7 a 14 & De más de 14 a 25 & De más de 25 a 50 & Mayor a 50 \\
\hline
\end{tabular}

Los valores registrados (CCP-UCR, 2004) y los códigos asignados para la TMI para el período 1995-2003 son:

\begin{tabular}{lccccccccc}
\hline AÑO & 1995 & 1996 & 1997 & 1998 & 1999 & 2000 & 2001 & 2002 & 2003 \\
\hline Cifras & 13,3 & 11,5 & 14,1 & 12,3 & 11,7 & 10,2 & 10,8 & 10,5 & 10,0 \\
Código & +1 & +1 & +1 & +1 & +1 & +1 & +1 & +1 & +1 \\
\hline
\end{tabular}

b. Para cada dimensión $k$ definida en el ICV:

$\mathrm{I}_{\mathrm{kt}}=$ Número de indicadores que estimaron la dimensión k-ésima en el año $t$ $\mathrm{V}_{\mathrm{ikt}}=$ valor $[-2,2]$ del i-ésimo indicador que estimó a la dimensión k-ésima para el año $t$ $\mathrm{C}_{\mathrm{kt}}=$ calificación de la dimensión k-ésima en el año $t$, definido como:

$$
C_{k t}=\frac{1}{4 I_{k t}}\left(\sum_{i=1}^{I_{k t}} V_{i k t}\right)+\frac{1}{2}
$$

Haciendo $\mathrm{I}_{\mathrm{kt}}=\mathrm{j}$ indicadores para la dimensión k-ésima en el año t, se puede obtener el ámbito de variación de $\mathrm{C}_{\mathrm{kt}}$. Si los $\mathrm{j}$ indicadores fueran calificados como regulares (igual a cero), entonces $\mathrm{C}_{\mathrm{kt}}=0,50$. Si los $\mathrm{j}$ indicadores fueran calificados como muy bueno $(+2)$, entonces $\mathrm{C}_{\mathrm{kt}}=1$. Por último, si los $\mathrm{j}$ indicadores fueran calificados como muy malo (-2), entonces $\mathrm{C}_{\mathrm{kt}}=0$. De esta forma, el ámbito de $\mathrm{C}_{\mathrm{kt}}$ va de 0 a 1 , propiedad deseada en un instrumento de calificación.

c. Finalmente, la estimación del ICV se obtuvo por medio del promedio de las calificaciones individuales de cada parámetro o dimensión ponderado por el número de indicadores que intervinieron en cada $\mathrm{C}_{\mathrm{kt}}$, simbólicamente,

$$
I C V_{t}=\frac{\sum_{k=1}^{K} C_{k t} I_{k t}}{\sum_{k=1}^{K} I_{k t}}
$$

Una vez obtenido el índice preliminar, se procedió a su validación.

\section{RESULTADOS}

\subsection{INDICADORES SELECCIONADOS}

Una vez revisada y consultada la bibliografía y habiéndose hecho un inventario de las estadísticas disponibles para el objeto de esta investigación y de su marco teórico previamente establecido, el cual se mostró en la figura 2 , se estableció la lista de indicadores que se muestra en el cuadro 1 con el fin de medir calidad de vida. 


\section{CUADRO 1}

COSTA RICA. INDICADORES DE CALIDAD DE VIDA POR DIMENSIÓN Y TEMA

\begin{tabular}{|c|c|c|}
\hline DIMENSIÓN & TEMA & INDICADOR \\
\hline \multirow{3}{*}{ Libertad } & Asociación y organización & Tasa de sindicalización \\
\hline & & Razón de los salarios medios de los hombres y las mujeres \\
\hline & Equidad & $\begin{array}{l}\text { Tasa de desempleo femenino como porcentaje de la tasa de desempleo } \\
\text { masculino }\end{array}$ \\
\hline \multirow{11}{*}{ Dignidad } & \multirow{2}{*}{ Trabajo } & Tasa de accidentes laborales \\
\hline & & Tasa de subempleo invisible \\
\hline & Acceso a la educación & Tasa neta de escolaridad en educación primaria \\
\hline & Seguridad social & Tasa de cobertura por seguridad social \\
\hline & \multirow{3}{*}{ Vida saludable } & Tasa de mortalidad infantil \\
\hline & & Esperanza de vida \\
\hline & & $\begin{array}{l}\text { Porcentaje de días de incapacidad por diagnóstico de causas de índole } \\
\text { psicológica }\end{array}$ \\
\hline & \multirow{4}{*}{ Ambiente seguro y saludable } & Concentración de $\mathrm{NO}_{2}$ en las áreas urbanas \\
\hline & & Concentración de $\mathrm{CO}$ en las áreas urbanas \\
\hline & & Casos registrados de Dengue \\
\hline & & Intoxicaciones por plaguicidas \\
\hline \multirow{8}{*}{ Seguridad } & \multirow{2}{*}{ Económica } & Tasa de desempleo \\
\hline & & Tasa de empleo en el sector informal \\
\hline & \multirow{2}{*}{ Personal } & Tasa de delitos contra la vida \\
\hline & & Tasa de delitos sexuales \\
\hline & Jurídica & Casos entrados en el Tribunal de la Inspección Judicial \\
\hline & \multirow{3}{*}{ Ciudadana } & Casos entrados por hurto \\
\hline & & Casos entrados por robo \\
\hline & & Casos entrados por secuestro \\
\hline \multirow{2}{*}{ Creatividad } & \multirow{2}{*}{ Investigación y desarrollo } & Coeficiente de inventiva \\
\hline & & Número de publicaciones nacionales \\
\hline Solidaridad & $\begin{array}{l}\text { Participación en asociaciones } \\
\text { de desarrollo comunal }\end{array}$ & Número de asociaciones de desarrollo comunal \\
\hline
\end{tabular}

\section{2. ÍNDICES AGREGADOS POR DIMENSIÓN}

Como se especificó en la justificación, una de las ventajas de los índices es que permiten resumir varios indicadores en una sola puntuación numérica, al mismo tiempo que, se conservan detalles específicos de cada indicador (Babbie, 1999). En el caso de los métodos de agregación de máximos y mínimos y de clases evaluativas aplicados en esta investigación, permitieron además de conservar los detalles de los indicadores, ver el comportamiento de cada una de las dimensiones de calidad de vida previamente definidas.

Luego de aplicar los pasos descritos en la sección 2.1.2.2 se obtuvieron los resultados que se muestran en el cuadro 2. 


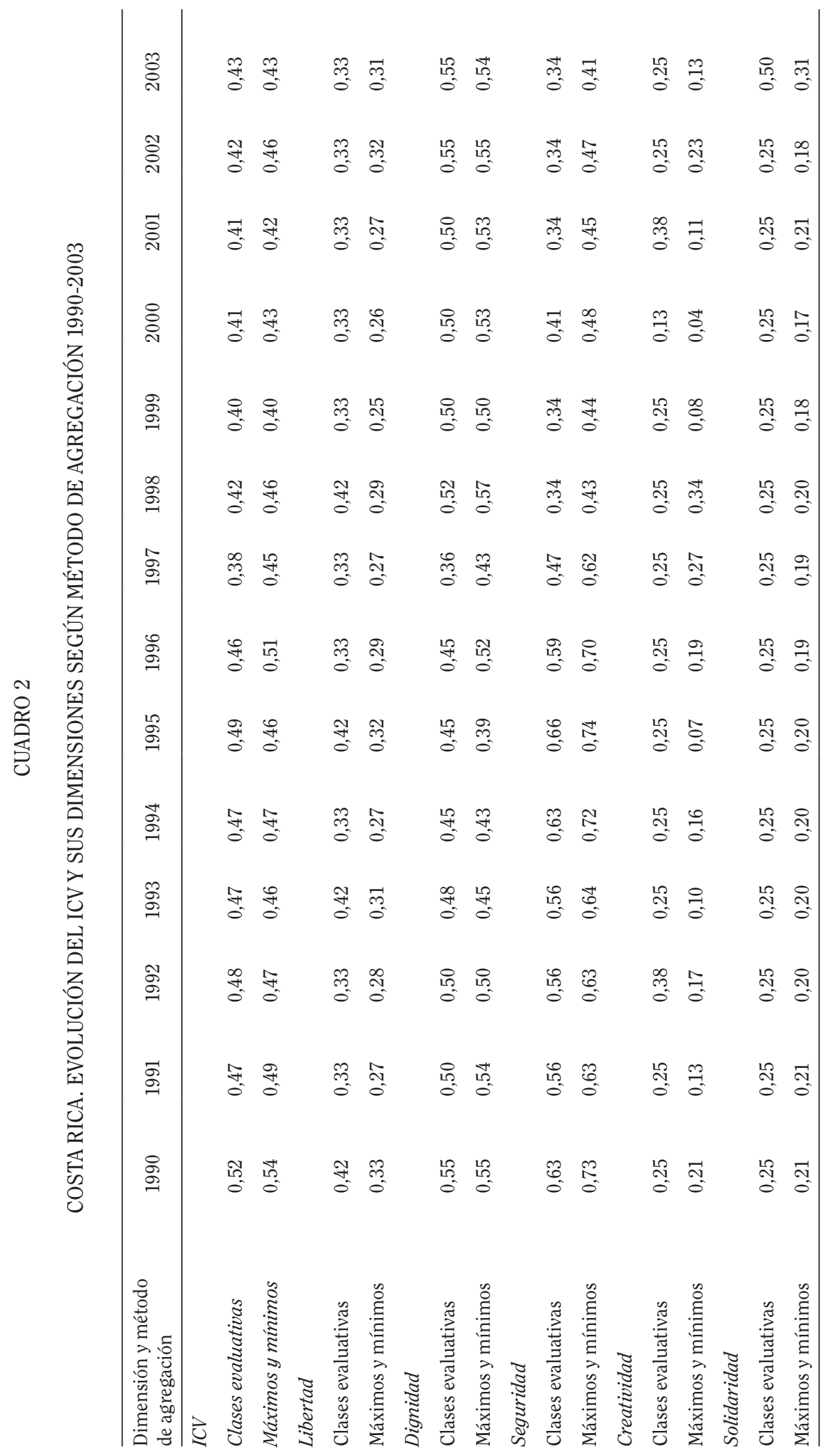


Partiendo con el análisis de la dimensión libertad, el indicador que incidió en forma más favorable entre los años 1990-2003 fue la razón de los salarios medios de los hombres y las mujeres, con una calificación promedio de 0,76 por el método de máximos y mínimos y de un +1 por clases evaluativas. Por otro lado, el indicador tasa de desempleo femenino como porcentaje de la tasa de desempleo masculina, tuvo un efecto desfavorable sobre esta dimensión, al extremo que para el período analizado en promedio según ambos métodos se obtiene una calificación mínima.

GRÁFICO 1
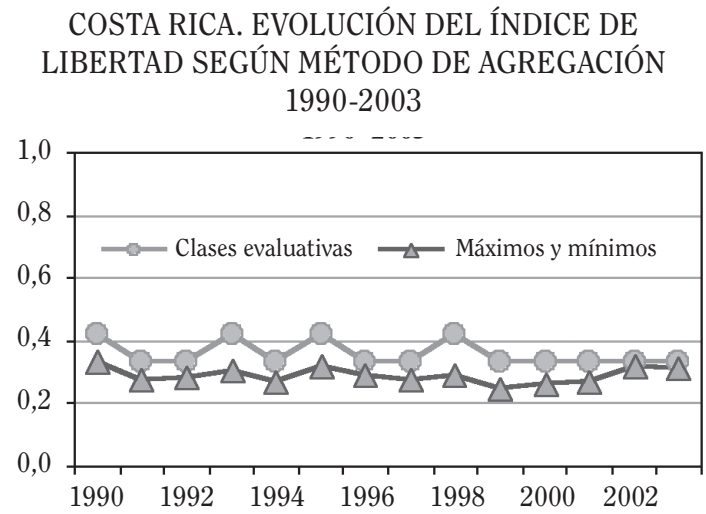

Al comparar los índices construidos a partir de los diferentes métodos de agregación, se determina fácilmente que para la mayoría de los años se obtuvieron mayores puntuaciones por el método de clases evaluativas. Asimismo, es posible observar que el índice calculado a partir de clases evaluativas mostró una mayor variabilidad (ver gráfico 1). El coeficiente de correlación de Pearson entre ambos índices fue igual a 0,613 (valor-p $=0,020$ ), lo que demuestra un alto grado de coincidencia en la tendencia de esta dimensión entre métodos de agregación.

Con relación a la dimensión dignidad, los indicadores que la afectaron en forma más favorable a lo largo del período analizado fueron la tasa de subempleo invisible (con una calificación promedio de 0,82 por el método de máximos y mínimos y de un +1 por clases evaluativas), la tasa de cobertura por seguridad social (con una calificación promedio de 0,84 por el método de máximos y mínimos y de un +2 por clases evaluativas) y la tasa de mortalidad infantil (calificación promedio de 0,82 por el método de máximos y mínimos y de un +1 por clases evaluativas). Por otro lado, los indicadores relacionados al tema de vivir en un ambiente seguro y saludable fueron los que obtuvieron una peor calificación en esta dimensión.

\section{GRÁFICO 2}

\section{COSTA RICA. EVOLUCIÓN DEL ÍNDICE DE DIGNIDAD SEGÚN MÉTODO DE AGREGACIÓN} 1990-2003

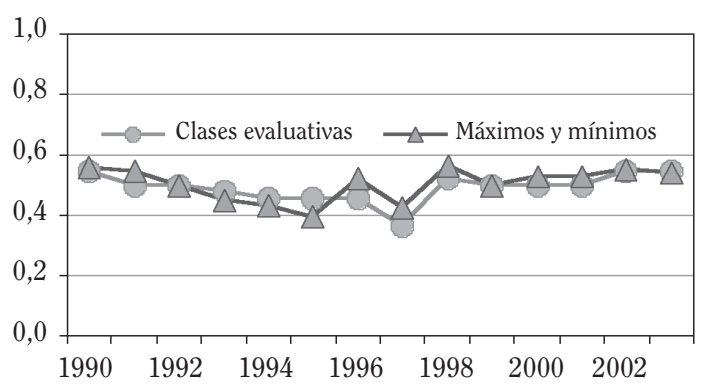

Los índices construidos a partir de los diferentes métodos de agregación presentaron una tendencia similar, mostrando una leve mejoría en el período analizado, alcanzando puntuaciones ligeramente superiores al punto medio (ver gráfico 2). El coeficiente de correlación de Pearson fue igual a 0,761 (valor-p = $0,002)$, demostrando asociación entre ambos índices. Uno de los elementos que contribuyeron a la similitud de los resultados entre ambos métodos de agregación fue sin lugar a duda el número de indicadores utilizado en su cálculo (11), esto sugiere que un buen número de indicadores para la medición de cada dimensión puede estar cercano a 10.

Ningún indicador relacionado con el tema de seguridad presenta un desempeño sobresaliente, teniendo una puntuación promedio cercana a 0,5 y con tendencia a declinar. Los indicadores que impactaron negativamente este índice fueron principalmente aquellos relacionados a la seguridad ciudadana.

Los índices construidos según los dos diferentes métodos de agregación presentaron una tendencia $y$ variabilidad similar, mostrando un deterioro en la seguridad de los habitantes 


\section{GRÁFICO 3}

COSTA RICA. EVOLUCIÓN DEL ÍNDICE DE SEGURIDAD SEGÚN MÉTODO DE AGREGACIÓN 1990-2003

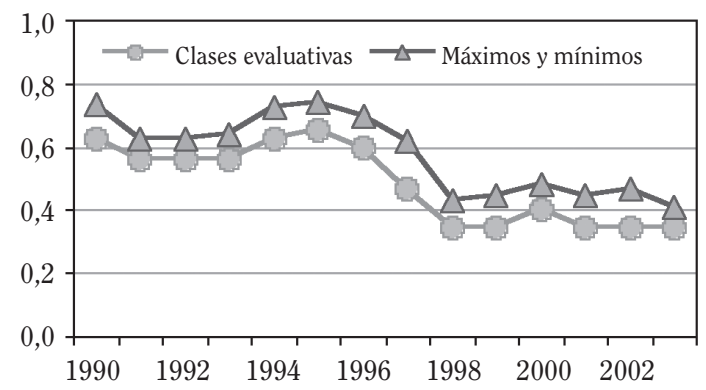

a lo largo del período analizado (ver gráfico 3). El grado de asociación determinado por el coeficiente de correlación de Pearson fue igual a 0,981 (valor-p $=0,000$ ), esto se traduce como una fuerte correlación entre ambos índices, nuevamente la coincidencia en los resultados presentados por ambos métodos puede ser atribuible en parte al número de indicadores utilizados en su cálculo (8).

En esta primera propuesta la creatividad estuvo medida únicamente por dos indicadores de investigación y desarrollo, quedando pendiente la inclusión de indicadores de cultura. En este ensayo no fue posible la inclusión de estos indicadores debido a que la información que posee el Ministerio de Cultura Juventud y Deportes no se encuentra sistematizada.

Debido a este escaso número de indicadores, el índice agregado de creatividad se mantuvo relativamente estable hasta el año 1999 para el método

\section{GRÁFICO 4}

COSTA RICA. EVOLUCIÓN DEL ÍNDICE DE CREATIVIDAD SEGÚN MÉTODO DE AGREGACIÓN 1990-2003

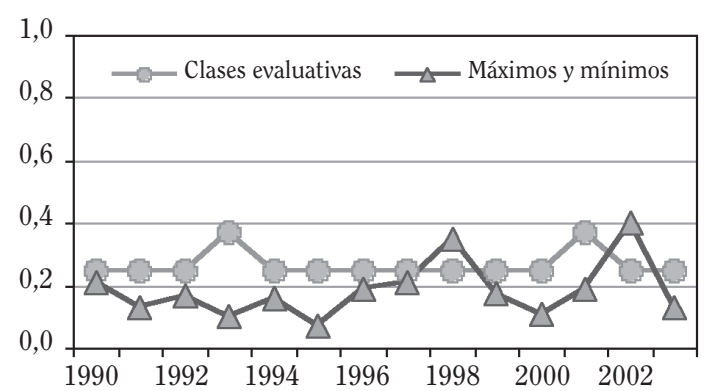

de clases evaluativas, por otro lado, el método de máximos y mínimos presentó una mayor sensibilidad ante cambios en las cifras (ver gráfico 4).

Preliminarmente, el índice de solidaridad estuvo constituido únicamente por la densidad de asociaciones de desarrollo comunal, esto debido a la poca disponibilidad de información relacionada al tema. Inicialmente, entre otros indicadores se evaluó la posibilidad de incorporar el número de voluntarios de la Cruz Roja, $y$ de bomberos voluntarios en el Instituto Nacional de Seguros, no obstante, en la actualidad no existe un registro de estas estadísticas. Debido a que el único indicador que conforma esta dimensión no presentó variaciones significativas en el período 1990-2002 tampoco lo hizo este índice (ver gráfico 5).

\section{GRÁFICO 5}

\section{COSTA RICA. EVOLUCIÓN DEL ÍNDICE DE SOLIDARIDAD SEGÚN MÉTODO DE AGREGACIÓN 1990-2003}

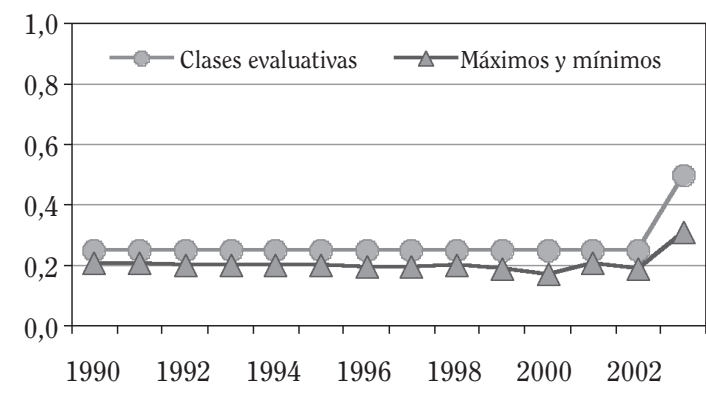

Luego de dar un vistazo general a los índices agregados para cada dimensión se concluye que únicamente los índices de dignidad y seguridad, poseen las propiedades estadísticas deseables (coincidencia en la tendencia por ambos métodos, baja variabilidad, número razonable de indicadores, etc.) para obtener un resultado confiable si se interpretan individualmente. Las limitaciones presentadas en las restantes dimensiones (libertad, creatividad y solidaridad) se dan principalmente por la escasa disponibilidad de la información estadística necesaria.

\section{3. ÍNDICE DE CALIDAD DE VIDA}

En el período comprendido entre 1990 y 2003, las puntuaciones obtenidas para el ICV 
son independientes del método de agregación utilizado, con un valor promedio de 0,45 , alcanzando niveles apenas aceptables de acuerdo al rango definido. El grado de asociación determinado por el coeficiente de correlación de Pearson fue igual a 0,777 (valor-p $=0,001$ ), esto se traduce como una fuerte correlación entre ambos índices. La variabilidad de ambos índices fue muy similar (una desviación estándar de 0,037 en el método de máximos y mínimos y 0,040 en el método de clases evaluativas).

\section{GRÁFICO 6}

\section{COSTA RICA. EVOLUCIÓN DEL ICV SEGÚN MÉTODO DE AGREGACIÓN} 1990-2003

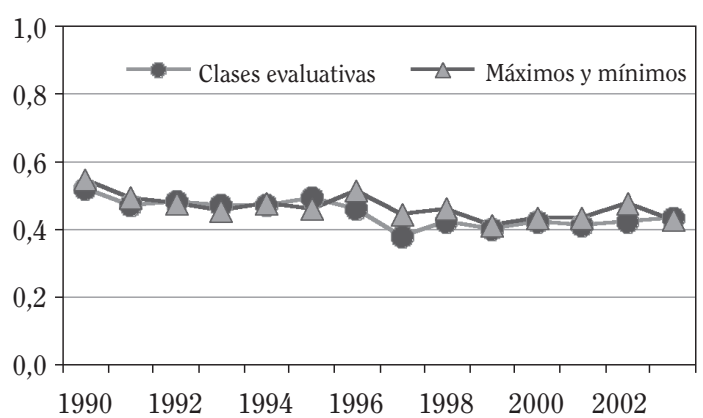

En el gráfico 7, se puede observar que independientemente del método de agregación empleado, la dimensión que incidió

\section{GRÁFICO 7}

COSTA RICA. PUNTUACIONES PROMEDIO ANUAL EN CADA DIMENSIÓN DEL ICV POR MÉTODO DE AGREGACIÓN 1990-2003

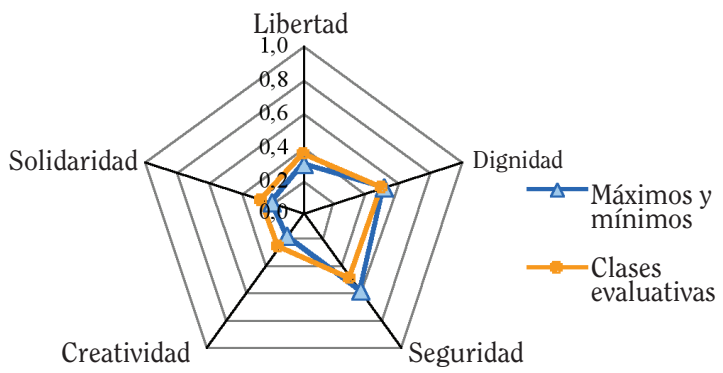

en forma más favorable en el ICV entre los años 1990-2003 fue la de seguridad, con una calificación promedio durante el período de 0,58 por máximos y mínimos y de 0,48 por clases evaluativas. Por otro lado, la dimensión que obtuvo la peor calificación fue la de creatividad con una puntuación promedio de 0,16 por máximos y mínimos y de 0,27 en el método de clases evaluativas.

\subsection{EVALUACIÓN DEL ÍNDICE}

\subsubsection{VALIDACIÓN INTERNA}

Para asegurar que el índice describe realmente el fenómeno de estudio es necesario ejecutar un proceso de validación. La lógica que se utiliza para validar un índice consiste en determinar hasta qué punto el indicador logra medir el fenómeno adecuadamente (Babbie, 1999).

La primera etapa para validar un índice es una validación interna, llamada análisis de item. El proceso consiste en determinar hasta qué grado cada uno de los ítem que conforman el índice está correlacionado con este; cuanto más altas sean las correlaciones tanto más válido será el indicador o índice (Babbie, 1999).

En el Cuadro 3, se muestran los coeficientes de correlación de Pearson con sus respectivos niveles de significancia para los indicadores seleccionados, con el fin de efectuar la validación interna. Se han señalado aquellas variables cuya asociación con los índices calculados es significativa al $15 \%$.

Los resultados obtenidos para los índices calculados por los dos métodos sugirieron que se deben definir diferentes indicadores para la medición del tema equidad y de la dimensión creatividad. Estos resultados también mostraron que para los indicadores seleccionados el método de clases evaluativas es mejor, ya que estos indicadores presentan correlaciones más altas con el ICV. 


\section{CUADRO 3}

\section{CORRELACIÓN DE LOS VALORES DE LOS INDICADORES CON LOS ICVS CALCULADOS POR MÉTODO DE AGREGACIÓN}

\begin{tabular}{|c|c|c|c|c|}
\hline \multirow[b]{2}{*}{ INDICADOR } & \multicolumn{2}{|c|}{ ICV POR MÁXIMOS Y MÍNIMOS } & \multicolumn{2}{|c|}{ ICV POR CLASES EVALUATIVAS } \\
\hline & $\begin{array}{c}\text { COEFICIENTE } \\
\text { DE } \\
\text { CORRELACIÓN } \\
\text { DE PEARSON }\end{array}$ & $\begin{array}{l}\text { SIGNIFICANCIA } \\
\quad(2 \text { COLAS })\end{array}$ & $\begin{array}{c}\text { COEFICIENTE } \\
\text { DE } \\
\text { CORRELACIÓN } \\
\text { DE PEARSON }\end{array}$ & $\begin{array}{l}\text { SIGNIFICANCIA } \\
\quad(2 \text { COLAS })\end{array}$ \\
\hline $\begin{array}{l}\text { TOTAL DE CORRELACIONES } \\
\text { SIGNIFICATIVAS AL } 15 \%\end{array}$ & & 14 & & 14 \\
\hline Tasa de sindicalización & 0,722 & $0,004^{*}$ & 0,792 & $0,001^{*}$ \\
\hline $\begin{array}{l}\text { Razón de los salarios medios de los } \\
\text { hombres y las mujeres }\end{array}$ & $-0,157$ & 0,591 & 0,090 & 0,759 \\
\hline $\begin{array}{l}\text { TDA femenina como porcentaje de la TDA } \\
\text { masculina }\end{array}$ & $-0,170$ & 0,560 & $-0,192$ & 0,511 \\
\hline Tasa de accidentes laborales & 0,654 & $0,011^{*}$ & 0,824 & $0,000^{*}$ \\
\hline Tasa de subempleo invisible & $-0,075$ & 0,800 & $-0,373$ & 0,189 \\
\hline $\begin{array}{l}\text { Tasa neta de escolaridad en educación } \\
\text { primaria }\end{array}$ & $-0,721$ & $0,004^{*}$ & $-0,700$ & $0,005^{*}$ \\
\hline Tasa de cobertura por seguridad social & $-0,502$ & $0,068^{*}$ & $-0,693$ & $0,006^{*}$ \\
\hline Tasa de mortalidad infantil & 0,602 & $0,023^{*}$ & 0,568 & $0,034^{*}$ \\
\hline Esperanza de vida al nacer & $-0,486$ & $0,078^{*}$ & $-0,565$ & $0,035^{*}$ \\
\hline $\begin{array}{l}\text { Porcentaje de días de incapacidad por } \\
\text { diagnóstico de causas de índole psicológica }\end{array}$ & $-0,012$ & 0,969 & $-0,131$ & 0,655 \\
\hline $\begin{array}{l}\text { Concentraciones de Dióxido de nitrógeno } \\
\text { en las zonas urbanas }\end{array}$ & 0,421 & $0,134^{*}$ & 0,349 & 0,221 \\
\hline $\begin{array}{l}\text { Concentraciones de Monóxido de carbono } \\
\text { en las zonas urbanas }\end{array}$ & $-0,544$ & $0,044^{*}$ & $-0,601$ & $0,023 *$ \\
\hline Casos registrados de dengue & $-0,482$ & $0,081^{*}$ & $-0,479$ & $0,083^{*}$ \\
\hline Intoxicaciones por plaguicidas & $-0,228$ & 0,432 & $-0,097$ & 0,741 \\
\hline Tasa de desempleo & $-0,372$ & 0,191 & $-0,604$ & $0,022 *$ \\
\hline Tasa de empleo en el sector informal & $-0,144$ & 0,624 & $-0,421$ & $0,134^{*}$ \\
\hline Tasa de delitos contra la vida & $-0,751$ & $0,002^{*}$ & $-0,793$ & $0,000^{*}$ \\
\hline Tasa de delitos sexuales & $-0,675$ & $0,009 *$ & $-0,692$ & $0,006^{*}$ \\
\hline $\begin{array}{l}\text { Casos entrados en el Tribunal de la } \\
\text { Inspección Judicial }\end{array}$ & $-0,506$ & $0,060^{*}$ & $-0,393$ & 0,164 \\
\hline Casos entrados por hurto & $-0,642$ & $0,014^{*}$ & $-0,717$ & $0,004^{*}$ \\
\hline Casos entrados por robo & $-0,735$ & $0,003^{*}$ & $-0,745$ & $0,002^{*}$ \\
\hline Casos entrados por secuestro & 0,118 & 0,691 & 0,355 & 0,213 \\
\hline Coeficiente de inventiva & $-0,085$ & 0,758 & $-0,053$ & 0,857 \\
\hline Títulos registrados en ISBN & 0,352 & 0,209 & $-0,124$ & 0,672 \\
\hline $\begin{array}{l}\text { Número de Asociaciones de Desarrollo } \\
\text { Comunal }\end{array}$ & $-0,095$ & $0,056^{*}$ & 0,085 & 0,772 \\
\hline
\end{tabular}

* Significativo al 15\%

\subsubsection{VALIDACIÓN EXTERNA}

Además del criterio de validez interna, es importante realizar una validación externa. Esta consiste en confrontar los resultados del índice con los resultados de un criterio externo, que mida lo mismo o algo similar a lo que se pretendió medir con el indicador o índice. En la presente investigación, como procedimiento de validación externa, se realizó el contraste 
en una escala temporal de la percepción de los ciudadanos de la evolución de la calidad de vida contra los cambios reflejados en el Índice de Calidad de vida.

Con el fin de evaluar la percepción del costarricense de la evolución de la calidad de vida en Costa Rica, la empresa Unimer incluyó en algunas ocasiones en sus Encuestas de Opinión la pregunta: en relación con hace 10 años ¿vivimos mejor hoy o hemos perdido "calidad de vida"? En el Gráfico 8, se muestran los resultados obtenidos en las entrevistas domiciliarias llevadas a cabo en mayo del 2000 y en enero del 2001.

\section{GRÁFICO 8}

COSTA RICA. PERCEPCIÓN DE LOS

ENTREVISTADOS ACERCA DE LA EVOLUCIÓN DE LA CALIDAD DE VIDA EN LOS ÚLTIMOS 10 AÑOS. ENCUESTAS DE MAYO 2000 Y ENERO 2001

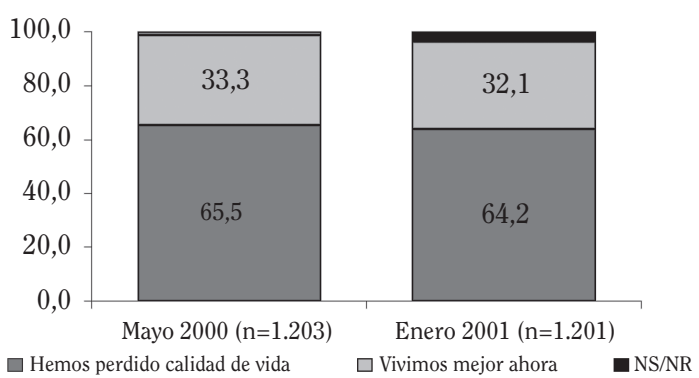

Fuente: Unimer RI, 2000 y 2001.

Es posible observar que en ambas encuestas, casi dos terceras partes de los entrevistados opinaron que en los últimos 10 años perdieron calidad de vida, en este caso los resultados dados por medio de los índices calculados respaldan la percepción de los ciudadanos. Sucede lo contrario con los resultados de la encuesta llevada a cabo por la misma empresa en Junio 2002, según la cual el porcentaje de los entrevistados que consideraban que se vivía peor que hace cinco años alcanzó el 40,7\%, mientras que los ICvs calculados mostraron una disminución en la calidad de vida (ver gráfico 9).

Estos resultados indican que los índices calculados a partir de estadísticas continuas,

\section{GRÁFICO 9}

COSTA RICA. PERCEPCIÓN DE LOS

ENTREVISTADOS ACERCA DE LA EVOLUCIÓN DE LA CALIDAD DE VIDA EN LOS ÚLTIMOS 5 AÑOS. JUNIO 2002

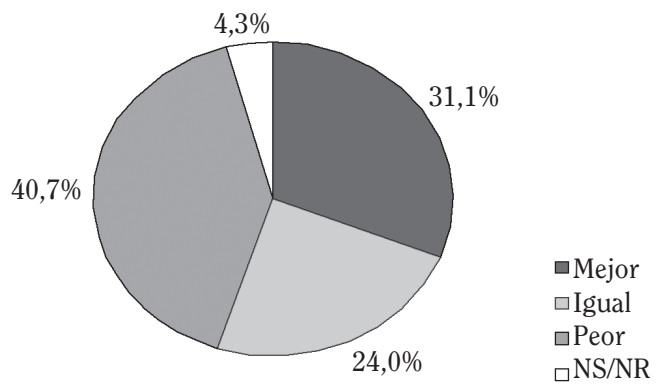

Fuente: Unimer RI, 2002.

permiten obtener conclusiones similares a las derivadas a partir de una encuesta de opinión que mida la percepción de los ciudadanos con relación a la evolución de la calidad de vida en un período de 10 años.

\subsubsection{EVALUACIÓN DEL SUPUESTO DE INDEPENDENCIA DE LAS DIMENSIONES DEL ICV}

Además de los procedimientos de validación interna y externa, en el caso específico de los índices calculados es importante validar el supuesto de independencia de las dimensiones que se hizo al utilizar un modelo aditivo para su agregación.

Con el fin de evaluar posibles asociaciones entre las dimensiones del ICV se calculó el coeficiente de correlación de Pearson. En el cuadro 4 se muestran los resultados obtenidos. Es posible observar que únicamente existe una asociación estadísticamente significativa al 5\% entre las dimensiones dignidad y seguridad calculadas por método de máximos y mínimos. La independencia encontrada en el modelo de clases evaluativas respalda la suposición que se hizo al elegir modelos aditivos para agregar estas dimensiones $y$ construir el ICV. 


\section{CUADRO 4}

CORRELACIONES ENTRE LAS DIMENSIONES DE CALIDAD DE VIDA SEGÚN MÉTODO DE AGREGACIÓN ${ }^{1 /}$

\begin{tabular}{|c|c|c|c|c|c|c|}
\hline DIMENSIÓN & & LIBERTAD & DIGNIDAD & SEGURIDAD & CREATIVIDAD & SOLIDARIDAD \\
\hline \multirow[t]{2}{*}{ Libertad } & $\begin{array}{l}\text { Máximos y } \\
\text { mínimos }\end{array}$ & 1,000 & & & & \\
\hline & Clases evaluativas & 1,000 & & & & \\
\hline \multirow[t]{2}{*}{ Dignidad } & $\begin{array}{l}\text { Máximos y } \\
\text { mínimos }\end{array}$ & $0,094(0,749)$ & 1,000 & & & \\
\hline & Clases evaluativas & $0,132(0,654)$ & 1,000 & & & \\
\hline \multirow[t]{2}{*}{ Seguridad } & $\begin{array}{l}\text { Máximos y } \\
\text { mínimos }\end{array}$ & $0,274(0,343)$ & $-0,537(0,048)$ & 1,000 & & \\
\hline & Clases evaluativas & $0,327(0,254)$ & $-0,358(0,208)$ & 1,000 & & \\
\hline \multirow[t]{2}{*}{ Creatividad } & $\begin{array}{l}\text { Máximos y } \\
\text { mínimos }\end{array}$ & $0,253(0,383)$ & $0,271(0,349)$ & $-0,023(0,938)$ & 1,000 & \\
\hline & Clases evaluativas & $0,164(0,575)$ & $-0,052(0,859)$ & $-0,118(0,687)$ & 1,000 & \\
\hline \multirow{2}{*}{ Solidaridad } & $\begin{array}{l}\text { Máximos y } \\
\text { mínimos }\end{array}$ & $0,345(0,228)$ & $0,155(0,598)$ & $-0,234(0,421)$ & $-0,031(0,915)$ & 1,000 \\
\hline & Clases evaluativas & $-0,175(0,549)$ & $0,327(0,254)$ & $0,323(0,261)$ & $-0,108(0,714)$ & 1,000 \\
\hline
\end{tabular}

* Significativo al 5\%

1/ Entre paréntesis se muestra la significancia

\section{CONCLUSIONES}

El ICV propuesto aquí es un intento por estimular el interés en la medición de la calidad de vida costarricense en una forma sistemática. Lejos de ser una propuesta definitiva es sólo un ejercicio preeliminar e inconcluso, el cual puede crecer con la retroalimentación con otros investigadores.

Dado que esta investigación tenía como objetivo la presentación de una primera propuesta para la medición de la calidad de vida costarricense, otro problema que fue ignorado es el desbalance que hay en el número de indicadores en las dimensiones. Óptimamente, este debería ser el mismo en cada uno. Los resultados obtenidos de la comparación de la evolución de los índices por el método de máximos y mínimos y el de clases evaluativas muestran que al aumentar el número de indicadores que se contemplan en el cálculo de cada dimensión, mejora su medición. Se sugiere que un buen número de indicadores podría estar entre 8 y 10 . No obstante, se requiere una mayor cantidad de indicadores por dimensión para hacer simula- ciones con diferentes tamaños de muestras $y$ así poder determinar el verdadero número óptimo de indicadores por dimensión.

Sin duda alguna, una de las prioridades para mejorar la validez de los índices aquí propuestos es la inclusión de indicadores anuales de participación en actividades políticas, participación en actividades culturales, de acceso a espacios y medios para la recreación $y$ esparcimiento y de voluntarismo. No obstante, ello requiere de una mejora en los registros de información estadística por parte de las instituciones encargadas. En el caso de los indicadores de cultura y deporte el MCJD y Ministerio de Planificación Nacional y Política Económica (MIDEPLAN) se encuentran trabajando en la sistematización de la información existente.

Otra recomendación es someter a evaluación la posibilidad de interacciones entre las cinco dimensiones definidas de calidad de vida, dado que tanto para el cálculo por el método de máximos y mínimos como por el de clases evaluativas se asumió independencia de los mismos. En el caso del primer método de 
máximos y mínimos no se cumplió la independencia estadística en todas las dimensiones, sin embargo, el modelo aditivo sí es útil para clases evaluativas.

El contraste de los resultados obtenidos por los índices calculados versus la percepción de los ciudadanos reflejados por medio de las encuestas de opinión, muestra una mayor coincidencia si se contempla la evolución en un período de 10 años que si se considera la variación en cinco años.

Entre las ventajas que pueden señalarse de los índices construidos es que al variar de 0 a 1 su interpretación es muy fácil. Además, dado que gran parte de las metas están basadas en referencias internacionales, este índice una vez mejorado podría ser aplicado en la evaluación y comparación de cifras entre países.

Los resultados de los índices preliminares mostrados en este documento permiten ver que desde el año 1990 hasta el 2003, la calidad de vida ha alcanzado niveles regulares (rondando el 0,5) y su tendencia es al deterioro. La disminución más importante es la mostrada en la dimensión seguridad, la cual pasó de tener una calificación aceptable en 1990 (0,7 por el método de máximos y mínimos) a un nivel regular en el $2003(0,4)$.

\section{REFERENCIAS}

Babbie, E. Fundamentos de la Investigación Social. International Thomson Editores. México D.F. México. 1999.

Brenes, H. "Propuesta de un índice para la medición de la calidad de vida en Costa Rica". Tesis Magister Scientae en Estadística. Universidad de Costa Rica. San José. Costa Rica. 2004.

CCP-UCR (Centro Centroamericano de Población de la Universidad de Costa Rica). Tasas demográficas básicas de Costa Rica 1941. <http://ccp.ucr.ac.cr/observa/ CRindicadores/tasas.htm > 2004 . [Consulta: 28 de junio, 2004].
FH (Freedom House). Freedom in the World Survey Methodology. <http://www. freedomhouse.org> 2003. [Consulta: 6 de julio, 2003]

González, C.M, Trejos, J.A. y Vargas, C.M. "Propuesta de un modelo multiplicativo para el índice aproximado de sostenibilidad". Memoria del seminario de graduación Licenciatura en Estadística. Universidad de Costa Rica. San José. Costa Rica. 1999.

Gutiérrez-Espeleta, E. E. "Indicadores de sostenibilidad: instrumentos para la evaluación de políticas nacionales". Revista de Ciencias Económicas. 1994. Vol. XIV, No.2. Págs. 37-50. Costa Rica.

. "Designing Environmental Indicators for Decision Makers". Proceedings Joint Conference of the International Association of Survey Statistician and the International Association for Official Statistician. Instituto Nacional de Estadística, Geografía e Informática. Aguascalientes, México.

INEC (Instituto Nacional de Estadística y Censos). "Población Ocupada en el Sector Privado en Establecimientos de 1 a 4 empleados (excluye servidores domésticos y pensionistas)". Tabulados especiales de la EHMP 1990 a 1999. Costa Rica.

"Cifras básicas sobre Fuerza de Trabajo Julio 2003”. Encuesta de Hogares de Propósitos Múltiples. Costa Rica.

- "Cifras básicas sobre Pobreza e Ingresos Julio 2003”. Encuesta de Hogares de Propósitos Múltiples. Costa Rica.

INEC y CCP-UCR."Población ocupada por sector institucional según tamaño del establecimiento y tamaño del hogar". Base de datos de la EHPM de los años 
2001 y 2002 del Centro Centroamericano de Población: <http://encuestas.ccp.ucr. ac.cr> [Consulta: 24 de noviembre 2003]

INS (Instituto Nacional de Seguros). Seguro de Riesgos del Trabajo: Indicadores de siniestralidad. <http://www.ins-cr.com> [Consulta: 29 de julio 2004]

MEP (Ministerio de Educación Pública). Tasas brutas de escolaridad en el Sistema Educativo. <http://www.mep.go.cr/ CuadroEscolaridadHistorico.html> [Consulta: 11 de noviembre 2003]

MINSALUD (Ministerio de Salud). Casos registrados de enfermedades de declaración obligatoria. San José. Costa Rica. 2004.

Nussbaum, M. "Mujeres e igualdad según la tesis de capacidades". Revista Internacional del Trabajo. 1999. Volumen 118. Número 3. OIT (Oficina Internacional del Trabajo). Ginebra. Suiza.

OdD-UCR (Observatorio del Desarrollo de la Universidad de Costa Rica). Disco Compacto Tendencias del Desarrollo Costarricense: series cronológicas 19852002. San José. Costa Rica. 2003.

OdD-UCR y MINAE. Indicadores del desarrollo sostenible de Costa Rica. San José. Costa Rica. 2002.

PEN (Proyecto Estado de la Nación). Estado de la Nación en Desarrollo Humano Sostenible: Sexto Informe 1999. Editorama. San José. Costa Rica. 2000.

PODER Judicial. Anuarios de Estadísticas Judiciales de los años 1990 a 2004.
Departamento de Planificación, Sección de Estadística. San José. Costa Rica. 1990-2004.

RAE (Real Academia Española). Diccionario de la Lengua Española. Vigésima segunda edición. Tomo I. Editorial Espasa Calpe, S.A. Madrid. España. 2001.

RPI (Registro de la Propiedad Industrial). Sistema Estadístico de Registros - Sistema de Patentes. Oficinas del Registro Nacional. [Consulta: 5 de enero 2004]. San José. Costa Rica. 2004 .

UNIMER RI. I Encuesta Nacional de Opinión del año 2000. <http://www.nacion.com/ln_ ee/encuestas/unimer/6-2000/encuesta. htm> [Consulta: 4 de noviembre 2002]

- I Encuesta Nacional de Opinión del año 2001. http://www.nacion.com/ln_ee/ encuestas/unimer/01-2001/Parte1.htm. [Consulta: 4 de noviembre 2002]

IV Encuesta Nacional de opinión del año 2002. <http://www.nacion.com/ ln_ee/encuestas/calidadvida1/encuesta. htm $>$ [Consulta: 4 de agosto 2002]

UNSD (Statistics Division of the United Nations Secretariat). 2003. Indicators on health. <http://unstats.un.org/ unsd/demographic/social/health.htm> [Consulta: 1 de abril 2003]

van Poll, R. "The Perceived Quality of the Urban Residential Environment". PhD-thesis. Center for Energy and Environmental Studies (IVEM). University of Groningen (RuG). The Netherlands. 1997. 


\section{ANEXO A}

\section{VALORES MÁXIMOS Y MÍNIMOS DEFINIDOS PARA CADA INDICADOR}

\begin{tabular}{|c|c|c|c|c|}
\hline INDICADOR & UNIDADES & MÍNIMO & MÁXIMO & $\mathrm{CASO}^{1}$ \\
\hline Tasa de sindicalización & Porcentaje & 10 & 85 & 1 \\
\hline $\begin{array}{l}\text { Razón de los salarios medios de los hombres y las } \\
\text { mujeres }\end{array}$ & Porcentaje & 100 & 200 & 2 \\
\hline $\begin{array}{l}\text { Tasa de desempleo abierto femenina como porcentaje de } \\
\text { la tasa de desempleo abierto masculina }\end{array}$ & Porcentaje & 100 & 150 & 2 \\
\hline Tasa de accidentes laborales & $\begin{array}{l}\text { Por mil trabajadores } \\
\text { asegurados }\end{array}$ & 50 & 200 & 2 \\
\hline Tasa de subempleo invisible & Porcentaje & 2 & 7 & 2 \\
\hline Tasa neta de escolaridad en educación primaria & Porcentaje & 85 & 100 & 1 \\
\hline Tasa de cobertura por seguridad social & Porcentaje & 70 & 90 & 1 \\
\hline Tasa de mortalidad infantil & Por mil nacidos vivos & 4 & 50 & 2 \\
\hline Esperanza de vida & Años & 60 & 85 & 1 \\
\hline $\begin{array}{l}\text { Porcentaje de días de incapacidad por diagnóstico de } \\
\text { causas de índole psicológica }\end{array}$ & Porcentaje & 1 & 20 & 2 \\
\hline Concentraciones de $\mathrm{NO}_{2}$ en las áreas urbanas & $\mu \mathrm{g} / \mathrm{m}^{3}$ & 25 & 40 & 2 \\
\hline Concentraciones de $\mathrm{CO}$ en las áreas urbanas & $\mu \mathrm{g} / \mathrm{m}^{3}$ & 6 & 9 & 2 \\
\hline Casos registrados de dengue & Por cien mil habitantes & 45 & 150 & 2 \\
\hline Intoxicaciones por plaguicidas & Por cien mil habitantes & 10 & 35 & 2 \\
\hline Tasa de desempleo & Porcentaje & 4 & 14 & 2 \\
\hline Tasa de empleo en el sector informal & Porcentaje & 10 & 45 & 2 \\
\hline Tasa de delitos contra la vida & Por cien mil habitantes & 150 & 750 & 2 \\
\hline Tasa de delitos sexuales & Por cien mil habitantes & 40 & 180 & 2 \\
\hline Casos entrados en el Tribunal de la Inspección Judicial & Por cien mil habitantes & 10 & 45 & 2 \\
\hline Casos entrados por hurto & Por cien mil habitantes & 75 & 350 & 2 \\
\hline Casos entrados por robo & Por cien mil habitantes & 100 & 500 & 2 \\
\hline Casos entrados por secuestro & Por millón de habitantes & 2 & 12 & 2 \\
\hline Coeficiente de inventiva & Por millón de habitantes & 5 & 40 & 1 \\
\hline Títulos registrados en ISBN & Por cien mil habitantes & 5 & 60 & 1 \\
\hline Número de Asociaciones de Desarrollo Comunal & Por diez mil habitantes & 2 & 15 & 1 \\
\hline
\end{tabular}

1/ $\quad$ En el caso 1 a mayor valor de la variable se supone una mejor calidad de vida, por el contrario en el caso 2 un aumento en el valor de la variable supone una reducción de la calidad de vida. 


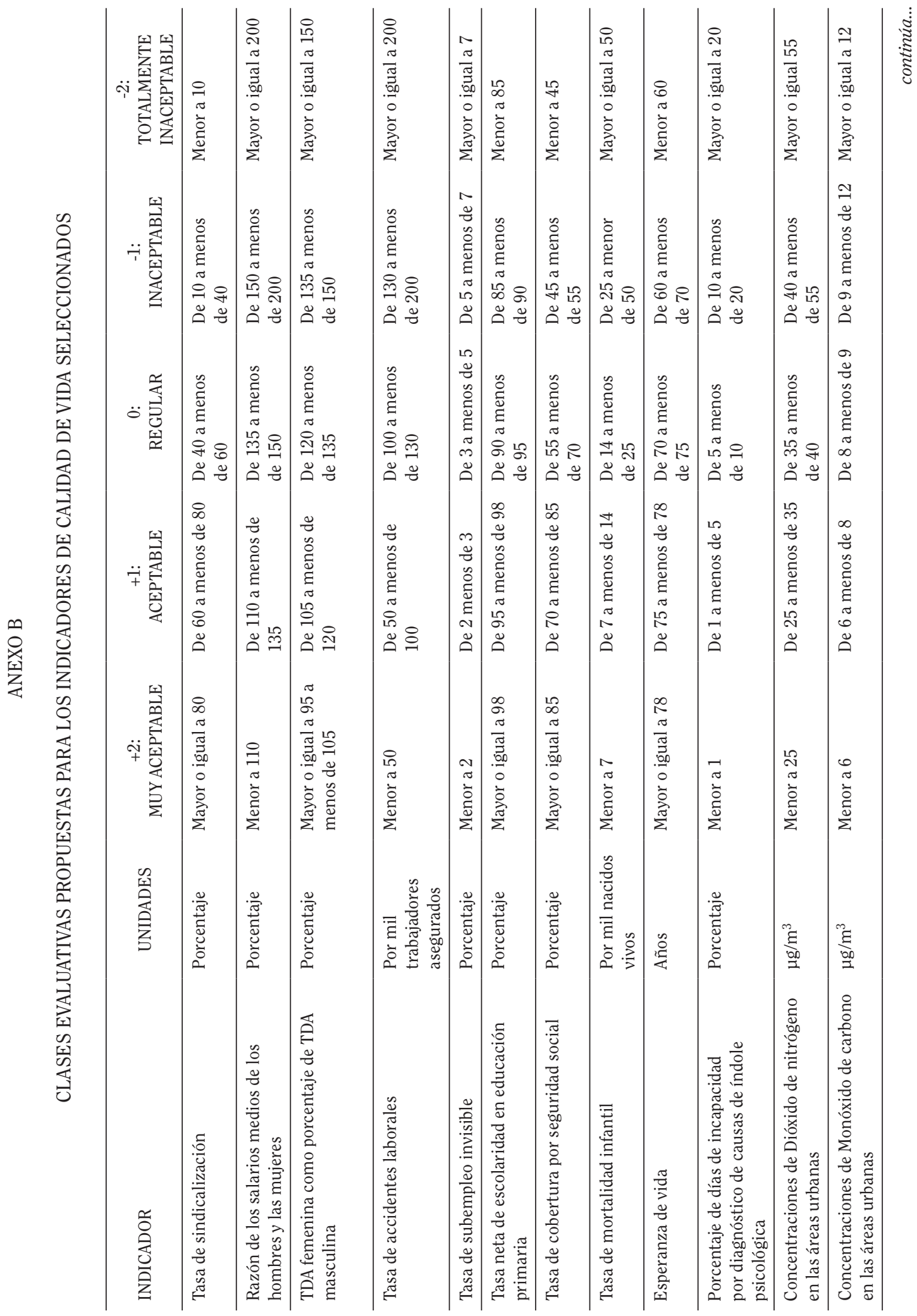




\begin{tabular}{|c|c|c|c|c|c|c|c|c|c|c|c|c|c|}
\hline 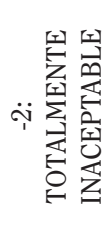 & 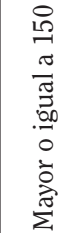 & 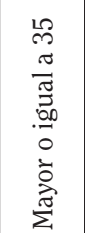 & 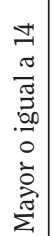 & 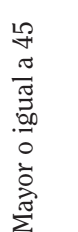 & 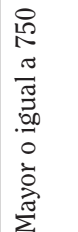 & 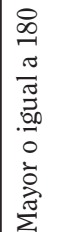 & 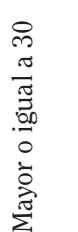 & 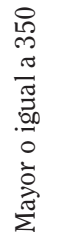 & 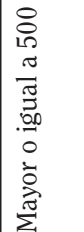 & 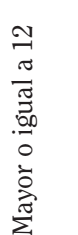 & 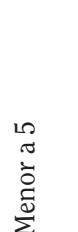 & 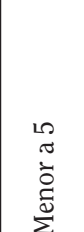 & 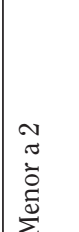 \\
\hline 空 & 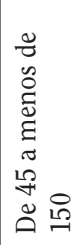 & 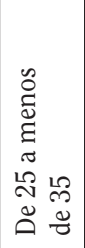 & 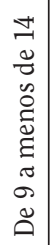 & 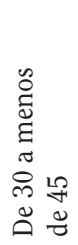 & 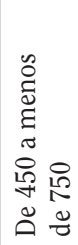 & 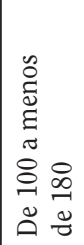 & 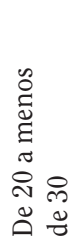 & 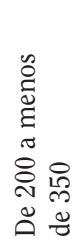 & 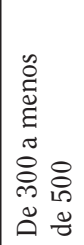 & 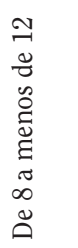 & 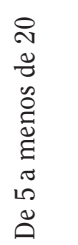 & 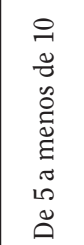 & 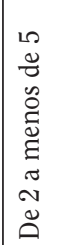 \\
\hline 。㟀 & 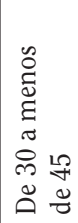 & 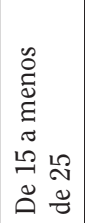 & 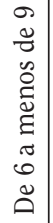 & 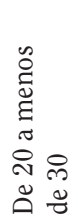 & 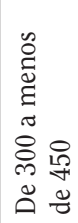 & 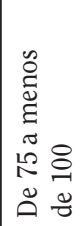 & 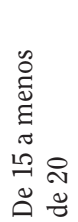 & 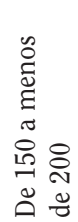 & 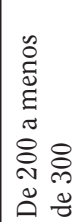 & 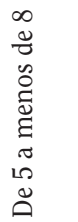 & 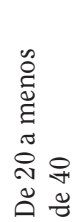 & 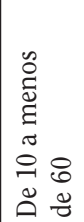 & 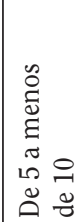 \\
\hline 芦窟 & 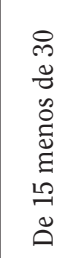 & 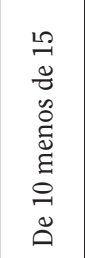 & 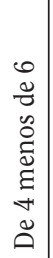 & 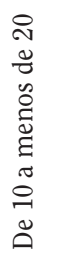 & 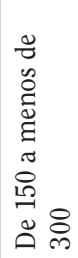 & 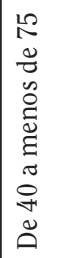 & 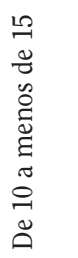 & 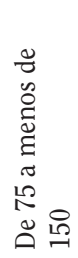 & 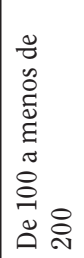 & 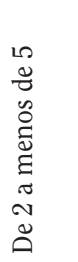 & 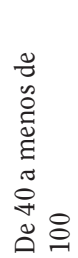 & 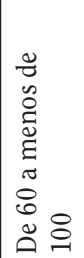 & 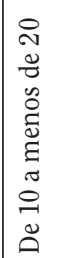 \\
\hline 突 & 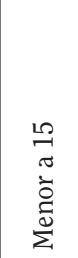 & 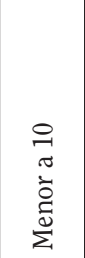 & 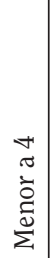 & 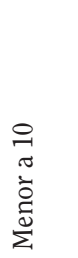 & 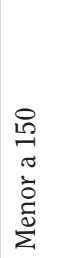 & 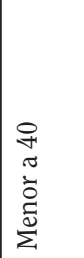 & 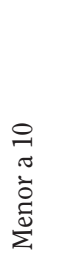 & 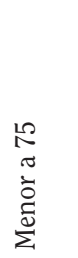 & 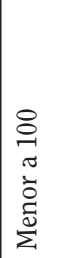 & 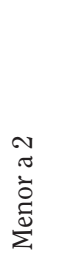 & 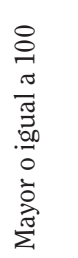 & 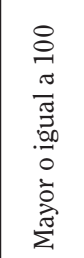 & 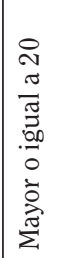 \\
\hline 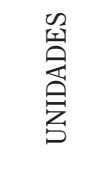 & 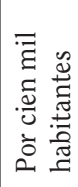 & 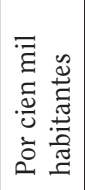 & 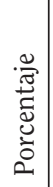 & 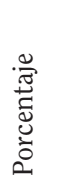 & 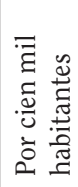 & 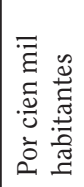 & 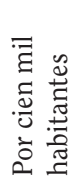 & 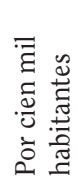 & 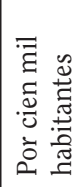 & 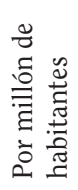 & 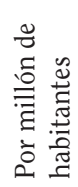 & 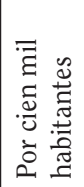 & 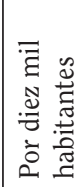 \\
\hline 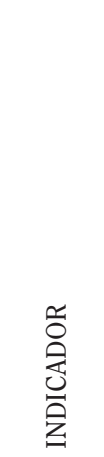 & 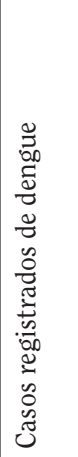 & 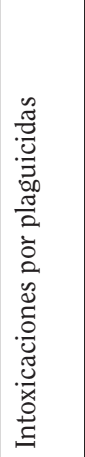 & 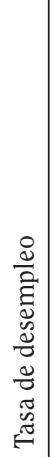 & 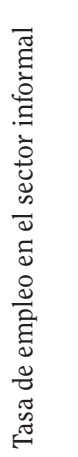 & 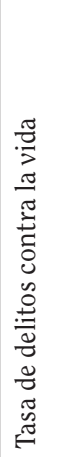 & 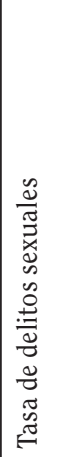 & 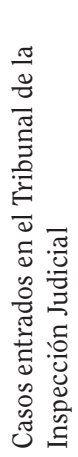 & 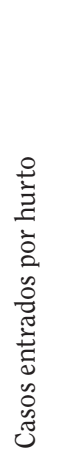 & 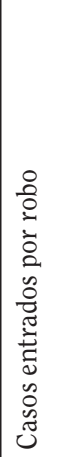 & 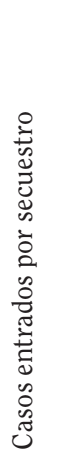 & 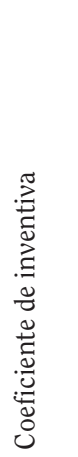 & 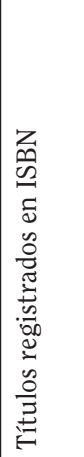 & 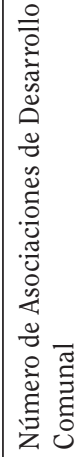 \\
\hline
\end{tabular}




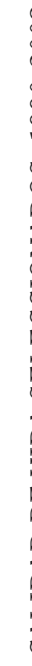

ชิ

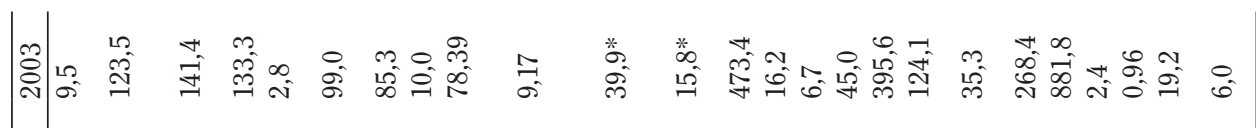

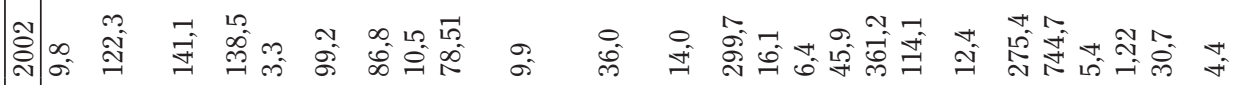

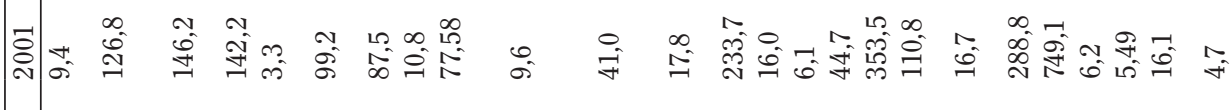

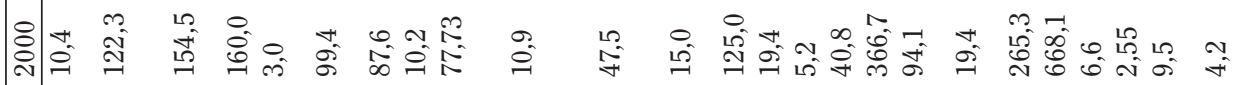

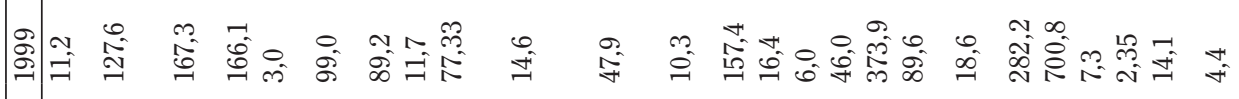

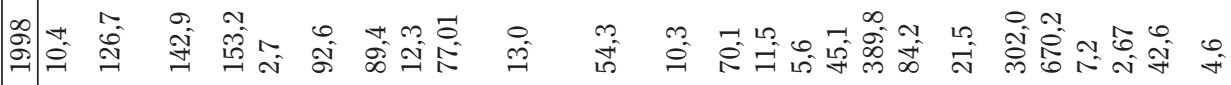

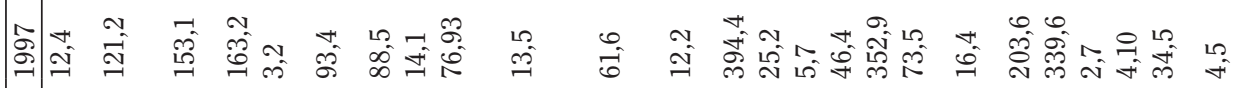

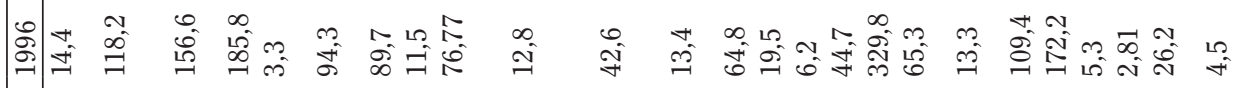

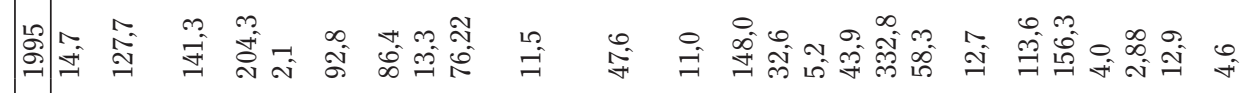

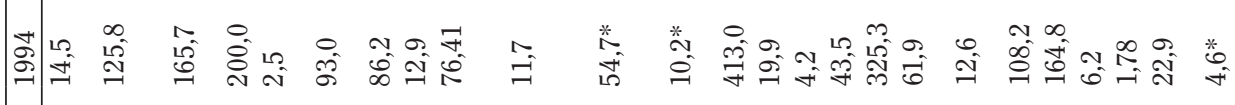

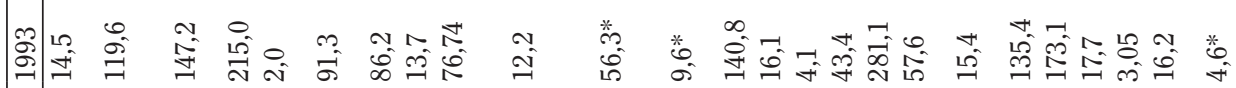

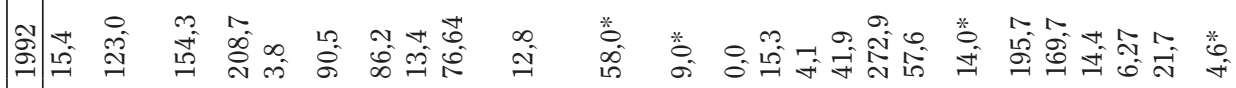

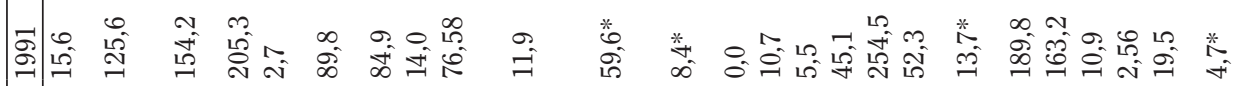

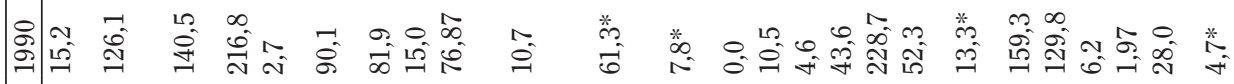

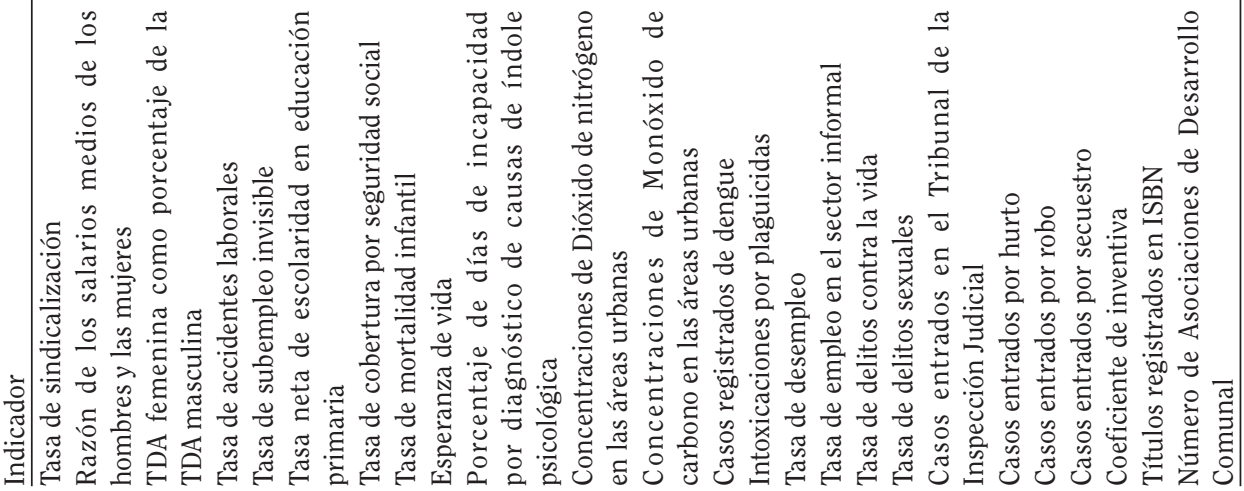

